\title{
Formación Inicial del Profesorado: Modelo Actual y Llaves para el Cambio
}

\section{Initial Teacher Education: The Current Model and the Keys to Change}

\author{
Denise Vaillant ${ }^{1, *}$ Carlos Marcelo ${ }^{2}$ \\ ${ }^{1}$ Universidad ORT, Uruguay \\ ${ }^{2}$ Universidad de Sevilla, España
}

\section{DESCRIPTORES:}

Profesorado

Formación inicia

Innovación

Modelo pedagógico

Cambio educativo

\begin{abstract}
RESUMEN:
En este artículo presenta las tendencias innovadoras en materia de formación inicial docente. El análisis se basa en referencias fundamentadas acumuladas por los autores en trabajos previos mediante una metodología cualitativa que orienta la revisión de la literatura a través de categorías apriorísticas de análisis. Hoy somos testigos del agotamiento del modelo de formación inicial docente frente a los retos planteados por las dos primeras décadas del siglo XXI. La literatura reconoce dos áreas primordiales en las que se puede reconocer la 'emergencia' de un modelo innovador. Por un lado, los cambios en la estructura y la organización de la formación y por otro la transformación del modelo pedagógico que da sustento a los programas de preparación inicial del profesorado. El trabajo que se comparte focaliza en los modelos pedagógicos y profundiza en casos de ruptura con el paradigma tradicional. Los antecedentes examinados se organizan en cuatro procesos innovadores que permitirían la mejora de la formación docente: la integración y la interdisciplinaridad basada en prácticas-clave; la consideración del aspecto emocional de la docencia; la promoción de la colaboración e intercambio y la valorización de los aprendizajes informales y de los procesos de autoformación.
\end{abstract}

\section{KEYWORDS:}

Teachers

Initial teacher

education

Innovation

Pedagogical model

Educational change

\section{ABSTRACT:}

This article presents the innovative trends in initial teacher education. The analysis is based on substantiated references accumulated by the authors in previous works using a qualitative methodology that guides the review of the literature through a priori categories of analysis. We are currently witnessing the exhaustion of the initial teacher training model in the face of the challenges posed by the first two decades of the 21st century. The literature identifies two primary areas in which the 'emergence' of an innovative model can be recognized. On the one hand, changes in the structure and organization of training and, on the other, the transformation of the pedagogical model that supports initial teacher education programs. The work that is presented focuses on pedagogical models and delves into cases of rupture with the traditional paradigm. The background experiences examined are organized into four innovative processes that would allow the improvement of teacher education: integration and interdisciplinarity based on key-practices; consideration of the emotional aspect of teaching; the promotion of collaboration and exchange; and the valorization of informal learning and self-training processes.

CÓMO CITAR:

Vaillant, D. y Marcelo, C. (2021). Formación inicial del profesorado: Modelo actual y llaves para el cambio. REICE. Revista Iberoamericana sobre Calidad, Eficacia y Cambio en Educación, 19(4), 55-69.

https://doi.org/10.15366/reice2021.19.4.003

*Contacto: vaillant@ort.edu.uy

ISSN: $1696-4713$

revistas.uam.es/reice 


\section{Introducción}

La formación inicial del profesorado ${ }^{1}$ de educación básica y media tiene el enorme compromiso de crear las condiciones para que los docentes que se integren en el sistema educativo posean las competencias necesarias para desenvolverse adecuadamente en el complejo mundo de la enseñanza. Sin embargo, se ha partido de enfoques culturales, curriculares, organizativos y prácticos que funcionaron en otros momentos históricos pero que hoy han perdido valor (Marcelo y Vaillant, 2018).

Los centros educativos actuales poco tienen que ver con las escuelas en las que estudiaban en el siglo XX los candidatos a convertirse en profesores. En 2017, Fernández Enguita publicó un libro con un título particular: "Más escuela y menos aula". El autor indicó que las escuelas actuales están concebidas según una estructura que fomenta el aislamiento, la repartición en disciplinas en los planes de estudios, la agrupación homogénea, la regulación del tiempo escolar a través de un estricto horario así como la evaluación a partir de pruebas estandarizadas. La descripción que hace el mencionado autor de la escuela es fácilmente trasladable a las instituciones de formación docente (Fernández Enguita, 2017).

El formato tradicional de preparación del profesorado registra serios signos de agotamiento ante los retos planeados por las dos primeras décadas del siglo XXI (Darling-Hammond, 2017). La manera en que se organiza el aprendizaje y la enseñanza, las características de aquellos que aprenden, así como las bases estructurales donde se asienta el conocimiento a trasmitir, han cambiado sustancialmente. Ya no parece suficiente la mejora del modelo pedagógico actual sino que se hace necesario una transformación profunda.

En un trabajo anterior que antecede este artículo (Marcelo y Villant, 2018), indicábamos que de acuerdo a Christensen y otros (2015) una innovación disruptiva ofrece una solución o respuesta nueva a quienes no tenían previamente opciones disponibles. En este artículo describimos algunas propuestas innovadoras y disruptivas para la formación inicial del profesorado. Se trata de experiencias que enseñan a pensar, comprender, sentir, conocer, buscar, resolver problemas, expresarse, respetar a los otros, trabajar colaborativamente y convivir en la diversidad (Bolick et al., 2019).

La formación del docente se ha movido permanentemente entre la tradición y la necesidad de innovación. Y es a esa tensión a la que nos referimos en los siguientes apartados. Tensión entre la conservación de las tradiciones y la búsqueda de nuevos enfoques y respuestas a las necesidades de un mundo cada vez más cambiante.

En los últimos veinte años, el número de actividades innovadoras en el campo de la formación se han multiplicado. Pero, a menudo, han tenido poca incidencia en los aprendizajes. Según Aguerrondo y Vaillant (2015), para poder llevar a cabo procesos de formación en el mundo actual, es necesario identificar las tendencias emergentes. Y son dos los ámbitos principales desde donde podemos encontrar estos enfoques emergentes: por un lado, los aspectos que tienen que ver con la estructura y organización de la formación docente y por otra parte con el modelo pedagógico que sustenta la propuesta de preparación inicial del profesorado

En este artículo nos hemos centrado en propuestas innovadoras en la formación inicial docente a partir de la revisión en bases de datos (ERIC, SCOPUS) y de antecedentes y referencias fundamentadas que figuran en un trabajo previo ya publicado (Vaillant y Marcelo 2018). Para delimitar el corpus de estudios que conforma la revisión de la literatura, se adoptó el marco metodológico diseñado por Wolfswinkel y otros (2011) y basado en las siguientes etapas: delimitación y búsqueda; selección e identificación de temas emergentes; interpretación de resultados y elaboración de conclusiones. El análisis e interpretación de datos se realizó a partir de cuatro categorías apriorísticas: la integración y la interdisciplinaridad basada en prácticas-clave; la consideración del aspecto emocional de la docencia; la promoción de la colaboración e intercambio y la valorización de los aprendizajes informales y los procesos de autoformación.

1 Aunque adherimos al uso no sexista del lenguaje, en este artículo empleamos el masculino genérico en atención a las normas de la RAE y por economía del lenguaje. Cabe señalar que el vocablo docente es utilizado como sinónimo tanto del vocablo maestro como del vocablo profesor y profesorado. 


\section{Transformar la malla curricular: Hacia la identificación de las prácticas clave}

Al revisar la malla curricular de los planes de formación del profesorado, es posible encontrar fragmentación y falta de coordinación entre diversos tipos de conocimiento. Es muy común que los contenidos, tanto disciplinares como pedagógicos que conforman el curriculum de la formación docente se presenten a los estudiantes mediante asignaturas separadas e inconexas (Feiman-Nemser, 2001, p. 1014). ¿Cómo superar ese divorcio?

Los profesores en formación suelen percibir que las normas de actuación y los conocimientos brindados en los centros educativos que concurren, tienen poco que ver con los conocimientos y las prácticas profesionales que observan cuando van a los centros educativos (Farrell, 2016). El curriculum de formación inicial tiende a descartar, por catalogarla como menos importante, la necesidad de integrar diversos conocimientos que son la base del trabajo práctico.

La formación docente debe incluir el conocimiento del contenido y el conocimiento didáctico del contenido, ya que las investigaciones han demostrado que hay una correlación positiva (Kleickmann et al., 2013). En este sentido, Berry y otros (2016) señalan que el desarrollo del conocimiento didáctico del contenido no finaliza en la etapa preparatoria del docente. Se trata de un proceso evolutivo que comienza en la formación inicial, se prolonga en la práctica docente y luego en el desarrollo profesional continuo. En ese sentido, se deberían ofrecer oportunidades de aprendizaje adecuadas y consistentes, que apoyen a los maestros y a los profesores en formación al desarrollo de ese conocimiento didáctico del contenido (Van Dijk, 2008).

Cuando se revisan las mallas curriculares de los programas de formación inicial docente, como ha hecho Flores (2016), se evidencia esta fragmentación. Asimismo, el análisis que hemos realizado en anteriores trabajos (Vaillant y Marcelo, 2015), evidencia tal fragmentación. Las propuestas curriculares presentan, por lo general, áreas disciplinares -como ser matemática e historia-, así como materias que tienen relación con la enseñanza, por ejemplo, psicología, pedagogía, sociología, filosofía. También se identifican áreas referidas a la didáctica de las disciplinas y a las prácticas de enseñanza.

Kennedy (2016) sostiene que, cuando la formación docente se desarrolla en las universidades, el conocimiento aparece fragmentado en tramos que suelen impartirse durante un curso semestral y un reducido número de créditos. El futuro profesor es considerado por su dominio disciplinar más que por sus competencias y capacidades en materia de enseñanza o por su expertise directa con la práctica que subyace al conocimiento. Si se valora al docente por el dominio de la disciplina más que por su enseñanza, obviamente la preparación del futuro profesor va a centrarse en el área disciplinar más que en los alumnos y el contexto de aula.

Las consecuencias del divorcio entre el conocimiento disciplinar y pedagógico, se reflejan en un docente que puede conocer los conceptos tanto de la disciplina como de la pedagogía, pero que se encuentra con serias dificultades para aplicar esos en situaciones de la práctica. Es un ejemplo de la separación entre teoría y práctica que Kitchen y Petrarca (2016) han analizado. Se trata de una propuesta interesante que permite observar diferentes enfoques en la formación del profesorado en base al énfasis que se le otorgue a la teoría, a la práctica y a la reflexión.

Kitchen y Petrarca (2016) destacan diferentes combinaciones posibles entre práctica-teoría y reflexión, en función de la integración de los diversos componentes de la propuesta curricular:

- Formación ofrecida a los candidatos a docentes a partir de una buena combinación de teoría, práctica y reflexión;

- Programas que valoran la práctica como punto clave de la formación docente y le otorgan a la teoría y a la capacidad de reflexión un papel secundario;

- Propuestas basadas en una perspectiva con un exceso de teoría y escasa práctica y reflexión; 
- Preparación del futuro docente que hace de la reflexión el eje principal, pero con escasa preocupación por la conceptualización o la práctica.

Los modelos que nos presentan Kitchen y Petrarca (2016) nos permiten situar en un eje de coordenadas las diferentes opciones extremas, dentro de las cuales caben modelos intermedios en los que priman en mayor o menor medida los diferentes componentes analizados.

\section{Figura 1}

El modelo de tres ejes en la formación del profesorado

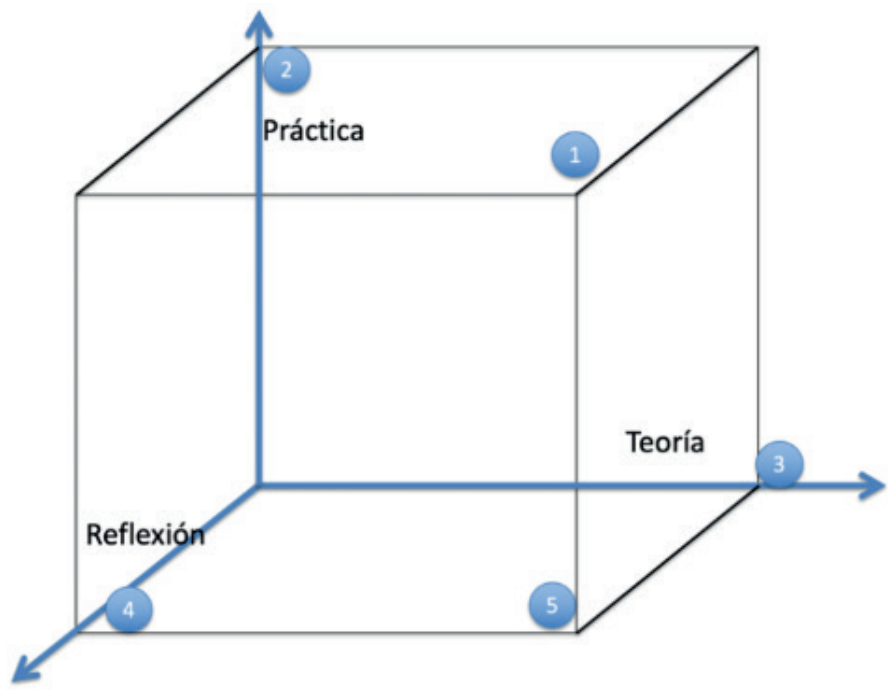

Nota. Elaborado a partir de Kitchen y Petrarca (2016).

Uno de los temas centrales en la agenda de la política de formación, refiere a la adecuada integración entre los saberes disciplinares que los docentes deben incorporar y las prácticas centrales en el ejercicio de la profesión (Darling-Hammond, 2017). Ello requiere desmantelar la separación tradicional que se produce en la preparación del profesorado entre las prácticas y los contenidos pedagógicos y disciplinares.

¿Cómo hacerlo? Grossman y otros (2009) han aportado un punto de vista interesante al organizar el currículo, para la formación inicial del profesorado, en relación a prácticas claves o core practices. Según la perspectiva de los autores, la transformación de una propuesta curricular basada en asignaturas a modelos enfocados en el ejercicio profesional, requiere que los formadores:

Trabajen en las actividades de clase y en los programas, para quebrar la tradicional separación entre la teoría y la práctica y entre contenidos y pedagogía;

Apoyen en la preparación del profesorado, en el desarrollo y perfeccionamiento de prácticas que son claves para la enseñanza.

De acuerdo con Grossman y otros (2009) las prácticas claves son las que habilitan a los futuros maestros y profesores a:

- Incluir en sus clases contenidos interdisciplinares, más allá del tipo de plan de estudios o de la metodología de enseñanza utilizada;

- Aprender más y mejor acerca de las características y necesidades de sus estudiantes y de las formas de enseñanza;

- Considerar la investigación y la evidencia para mejorar el desempeño de los estudiantes;

- Contemplar la complejidad y la integridad de la enseñanza. 
Se trata de centrar el aprendizaje en "centros de interés", en núcleos de contenidos prácticos que son habituales en la actividad docente (McDonald y otros, 2013). Algunas de estas prácticas claves podrían ser: dirigir una sesión de lectura guiada, explicar claramente para facilitar la comprensión de los estudiantes, interactuar con los padres, crear entornos de aprendizaje eficaces. Esta orientación que plantean Grossman et al. (2009) no es sólo una propuesta teórica, sino que es un proyecto que ha venido aplicando en diferentes áreas de conocimiento (Dinkelman y Cuenca, 2020; Dotger, 2015; Grosser-Clarkson y Neel, 2020; McDonald et al., 2013; Ward, 2021).

La noción de las prácticas claves nos lleva a comprender que la preparación de maestros y profesores debe centrarse en los problemas o en los casos y no solo en los contenidos. Estas situaciones que surgen durante la formación de los docentes permiten la integración de los conocimientos disciplinares, de los saberes pedagógicos, de las prácticas y de la reflexión.

Una conceptualización de la formación docente basada en las prácticas claves coloca al practicum como un elemento fundamental de un programa profesional (Mérida-Serrano et al., 2020). Al mismo tiempo, revierte la división tradicional entre práctica y cursos dentro del programa. Existen algunos ejemplos internacionales que van en el sentido de superar ese divorcio entre teoría y prácticas. Entre éstos figura trabajo desarrollado por Lampert y otros (2013) a través del cual los docentes en formación se involucran en la observación, en la planificación y en actividades modélicas que constituyen buenos ejemplos para comprender e impulsar procesos de enseñanza con buenos resultados para el aprendizaje de los estudiantes. Se trata de que los profesores en formación se ejerciten en el diseño de actividades de enseñanza.

Por su parte, Janssen y otros (2014) desarrollaron una propuesta de prácticas clave para docentes en formación en los laboratorios de biología, en las universidades de Leiden, Amsterdam y Arizona. También es posible identificar el trabajo de Dotger, en 2015, quien propone procesos de simulación, inspirados en la carrera de medicina para la preparación del profesorado. Esa experiencia se desarrolló en la Universidad de Syracuse, a partir de procesos de simulación en situaciones prediseñadas, en las cuales participaban actores reales y docentes en formación.

Como vemos, colocar en el centro de la formación inicial docente las prácticas clave, lleva necesariamente a un replanteamiento del currículum formativo, pero también de los espacios de aprendizaje. Las aulas tradicionales entendidas como "cartones de huevos" (Lortie, 1975) han de dar paso a ambientes más flexibles y abiertos, donde formadores de diferentes especialidades compartan espacios y tiempos para facilitar la adquisición por parte de los estudiantes de un conocimiento integrado. Pero para ello seguramente será necesario una reestructuración de los espacios y ambientes de aprendizaje tal como plantea Daniels en la Universidad de Oxford (Daniels et al., 2017). Esos autores han estudiado la forma como los nuevos espacios escolares permiten el desarrollo de nuevas metodologías y culturas de enseñanza. Pero es la intención hacia un enfoque de aprendizaje profundo lo que realmente importa y tanto mejor si el diseño lo facilita. Y en este sentido la intención hacia la integración y la interdisciplinaridad en los conocimientos y contenidos en la formación inicial docente debería abrirse paso entre una cultura academicista y en cierto modo obsoleta que persiste en mantener el status quo tradicional.

\section{Cambiar la formación academicista: Hacia la valoración de la dimensión socioemocional}

En uno de nuestros libros (Marcelo y Vaillant, 2009), planteamos que una de las necesidades centrales a atender en los programas de apoyo a los profesores, refiere a la dimensión socioemocional. A menudo olvidamos que la preparación de maestros y profesores no solo les proporciona conocimientos sino también una forma de ser ciudadanos y personas. Es necesario reconocer que gran parte de los problemas de desequilibrios, desmotivación y abandono que se producen en la educación, en muchos países, están relacionados con la escasez de adaptación, de madurez personal y emocional, así como de estrategias para afrontar a situaciones personales difíciles. Es llamativo que en muchos países las tasas de abandono de la profesión docente por parte de los profesores noveles sean muy altas. Los estudios indican que entre el 40 y el $50 \%$ de los profesores noveles aban- 
donan la enseñanza entre los primeros 5 años en Estados Unidos; en Australia el 10\%; en Suiza el 13\%; en Bélgica el 45\%; 29\% en Holanda, en Israel el 35\% o el 40\% en Chile (Aarts et al, 2020; Ávalos, 2014; Zavelevsky y Lishchinsky, 2020).

Korthagen (2014) es uno de los autores que más ha estudiado las propuestas de formación que incorporaran la dimensión socioemocional. El autor critica las limitaciones que tiene la sola consideración de la teoría como base para la enseñanza. Por esa razón es fundamental incorporar en la formación de un docente, instancias de reflexión acerca de sus emociones y sentimientos. Un modelo centrado solamente en la dimensión competencias, puede resultar demasiado limitado (Korthagen y Nuijten, 2018).

Korthagen (2016) también propone el llamado modelo cebolla para apoyar la reflexión del docente. La metáfora de las capas de la cebolla permite identificar seis niveles diferentes de interacción de los docentes. Mientras que algunos son públicos, otros son privados. Hay determinados niveles que son profesionales y otros personales. En el primer nivel, los maestros y profesores pueden reflexionar sobre un alumno o una clase en especial. En el segundo, acerca del comportamiento educativo del estudiante y, en el tercero, sobre sus competencias. En el nivel cuatro, el análisis se profundiza cuando se incorpora la reflexión sobre las creencias subyacentes. En el nivel cinco, se añaden las relaciones acerca de cómo cada persona percibe su propia identidad personal o profesional. Finalmente, en el sexto nivel, es posible reflexionar sobre el lugar y la misión de cada uno en el mundo.

La propuesta de Korthagen se aplica en diversas universidades de Holanda, como el Instituto de Educación de Utrech (Attema-Noordewier et al., 2012) y en los Estados Unidos, en la Southern Oregon University (Adams et al., 2013). El enfoque destaca la necesidad de que el docente tome consciencia de su identidad profesional, es decir, de sus ideales, sus finalidades y su propia imagen. También debe contemplar la comprensión de sus debilidades y fortalezas, el conocimiento de sí mismo para estar mejor preparado para afrontar situaciones desafiantes a nivel personal y profesional. Este enfoque que propone incluir la dimensión socioemocional en la formación del docente tiene sus problemas ya que puede crear resistencias, básicamente a nivel de los formadores de maestros y profesores (Russell, 2018).

La tendencia internacional valoriza la concepción de la práctica dentro del curriculum de la formación inicial docente, acompañada inevitablemente por un proceso de análisis, revisión y reflexión de esa práctica. Esta no es una idea nueva. Ya fue propiciada, entre otros, por Donald Schön (1983) quien enfatizó en la necesidad de aprender a través de la práctica (experiencia), mediante una reflexión que habilite a los profesionales a analizar, discutir, evaluar y cambiar su propia práctica.

La reflexión no es fortuita, sino que hay que preparar a los maestros y a los profesores para que puedan desarrollar madurez personal y profesional. A veces, es necesario dejar de lado el curriculum formal para poner la lupa en las experiencias de los estudiantes de formación docente. Y en ese proceso, el papel de los formadores de docentes es clave ya que son ellos quienes deciden cuánto tiempo dedican a esos procesos (Lunenberg et al., 2014).

Las narrativas constituyen una de las formas que permiten reflexionar acerca de los aspectos que conforman la identidad profesional docente. Éstas fomentan la reflexión, ya que impulsan a que el docente en formación se pregunte quién es y en qué se está convirtiendo como profesional y como persona. Cuando los maestros y profesores se involucran en un dialogo acerca de sus experiencias, utilizan anécdotas, imágenes y metáforas para contar una historia.

Kelchtermans (2014) sostiene que los docentes son narradores de historias, que pertenecen al ámbito de privado y de lo informal. En diferentes países se ha acumulado experiencia para incorporar, en la formación inicial docente, la narrativa de la práctica. Finlandia es uno de los ejemplos más conocidos en el uso de narrativas en la formación inicial del profesorado. Estola y otros (2014) detallan la experiencia en Finlandia, a partir de una serie de preguntas que refieren a la identidad de la persona y del docente, a sus motivaciones, a sus finalidades y a sus concepciones de vida.

En Australia (McGraw, 2014) también se ha empleado la narrativa como metodología en la preparación del profesorado a partir de cuatro procesos fundamentales: descripción; información, confrontación y reconstrucción. Además de la reflexión, la metodología propuesta, utiliza el dibujo 
para evocar ideas, pensamientos y sentimientos. Estos dibujos ayudan a las personas a interpretar sus concepciones. Las narrativas habladas, escritas y dibujadas revelan mucho acerca de los aspectos éticos, pedagógicos y relacionales de la profesión docente. En Singapore (Loh, 2014) y en Japón (Howe y Masahiro, 2014) también se ha utilizado la narrativa como un componente clave del programa de formación inicial docente. En esos casos, la narrativa ha sido útil, no sólo para desarrollar una experiencia autobiográfica y de autoconsciencia del docente, sino para establecer nexos significativos de relación con la teoría que los estudiantes en formación han de conocer y comprender.

La narrativa y la autobiografía como vía de autoconsciencia, de reflexión y de aprendizaje individual y colectivo también han estado presentes en los investigadores de América Latina. Así, existen diversos estudios realizados en el marco de la Associação Brasileira de Pesquisa. También la narrativa ha sido impulsada por la Red BioGrafia, Red científica de investigación biográfica en educación América latina-Europa. Esa red ha desarrollado iniciativas para ofrecer una mirada personal, subjetiva, comprensiva del ser docente desde una perspectiva basada en el análisis y comprensión de la memoria (Murillo Arango, 2015). Las narrativas a las que nos hemos referido anteriormente toman sentido en la medida en que propician la reflexión de los docentes, ya sean en formación inicial o continua. Pero promover la reflexión en las personas no un proceso automático y lineal. De hecho, podríamos decir que la reflexión está más presente en los documentos y programas oficiales que en las prácticas docentes de los formadores de formación inicial docente.

Russell (2014) fue uno de los pioneros en tratar de transformar la formación inicial docente en Canadá a través de los procesos de reflexión. Después de más de treinta años como formador, propone una serie de principios que, desde su punto de vista, deberían guiar la práctica reflexiva del profesorado en formación y de los docentes en ejercicio:

- Relaciones e intercambios formativos basados en la confianza mutua;

- Reconocimiento de las características únicas de cada persona que enseña;

- Consideración por los conocimientos y experiencias previos que docentes y formadores han adquirido en su paso como alumnos por las escuelas;

- Desarrollo de la reflexión y la metacognición;

- Modelamiento explícito y demostración por parte del formador;

- Aprendizaje por la experiencia además de lecturas y consultas a autores;

- Inclusión de la reflexión a lo largo de todo el proceso de formación.

Como vemos, la preocupación por indagar sobre los procesos de construcción de la identidad personal y profesional docente surge ante el reconocimiento de que la docencia no sólo es una actividad cognitiva y racional. Que al enseñar generamos emociones que nos sirven para expresarnos, para comunicarnos y ser parte de otros; que los docentes sufren y se alegran con sus alumnos, y que estas emociones han de estar presentes en los procesos de formación.

Incorporar la dimensión emocional en la formación inicial es todo un desafío disruptivo en particular para los formadores. Hemos de entender que el docente que formamos es un individuo que debe tener las herramientas necesarias para comprenderse y comprender situaciones de desafío a nivel personal, de búsqueda de metas, para involucrarse o alejarse emocionalmente de los contextos escolares que pueden crear malestar y estrés.

\section{El aprendizaje horizontal: Colaboración e intercambio}

En nuestros anteriores trabajos (Vaillant y Marcelo, 2015) hemos formulado críticas a los modelos de formación por su carácter tradicional y transmisivo. Como hemos expresado anteriormente, la formación docente presenta con frecuencia, una débil relación entre las prácticas de enseñanza y los cursos, seminarios y talleres impartidos en una universidad o instituto de formación docente. 
La fragmentación es el signo distintivo. Son pocos los formadores que practican lo que enseñan y escasas las ocasiones para el análisis y reflexión sobre la práctica (Marcelo y Vailant, 2018). Los informes internacionales apuntan a esa dirección. La formación se organiza en torno a estrategias en las que priman la participación individual del docente en cursos, talleres o seminarios. El informe TALIS de 2018 (OCDE, 2019) evidenciaba que el 76\% de los profesores encuestados ha participado en al menos un curso o taller en el último año. Frente a este porcentaje, un $40 \%$ de docentes ha intervenido en redes de profesores vinculadas con el desarrollo profesional docente.

Entre las iniciativas que buscan favorecer el trabajo colaborativo entre docentes, se destacan las comunidades de aprendizaje profesional y las redes de profesionales de la enseñanza. El trabajo colaborativo a través de esos escenarios requiere de docentes motivados, empoderados y con capacidad de liderazgo e innovación. En esa dirección, Patton y Parker (2017) se interrogan acerca de cómo fomentar desde la formación inicial, el desarrollo de espacios que favorezcan el trabajo colaborativo y las redes profesionales docentes.

El aprendizaje del docente, puede producirse de forma relativamente autónoma, personal e informal (Hoekstra et al., 2007). Pero la formación también puede entenderse como un proceso en el que el docente aprende de forma aislada, sino en colaboración, como una experiencia que ocurre al interactuar con los contextos o con los ambientes con los cuales los individuos se vinculan activamente.

El aprendizaje de los docentes puede ocurrir en cualquier momento, lugar y compañía. Se produce en contextos formales, pero se desarrolla cada vez más en situaciones informales (Kyndt et al., 2016). El aprendizaje en las redes sociales, ya sean de ocio o profesionales, se genera porque nos conectamos y participamos en comunidades de práctica. Desde hace décadas, desde la academia, se insiste en la necesidad de modificar las estrategias formativas y quebrar el aislamiento docente (Marcelo y Vaillant, 2009). El profesorado pasa su tiempo en soledad, no solo al momento de la planificación, sino en el trabajo de aula (Hargreaves, 2005). La evidencia indica que la mejora de la práctica docente requiere de las interacciones entre colegas (Stoll y Louis, 2007). Las comunidades de aprendizaje profesional surgen para combatir el aislamiento y promover el apoyo y la cooperación entre colegas (Hadar y Brody, 2010).

La dimensión comunitaria de la profesión docente es fundamental y parte del compromiso mutuo en el desarrollo de aprendizajes compartidos. La preparación inicial de profesorado debería incluir ese formato y orientarse hacia el intercambio de problemáticas profesionales como eje de la formación. Los futuros maestros y profesores podrían así participar desde las etapas iniciales de su preparación en comunidades de aprendizaje profesional.

Las formas de aprender del docente suelen no ser tenidas en cuenta en las propuestas de formación (Gómez-Hurtado et al., 2019). Boyle y otros (2004) indican que las actividades de formación se integran con frecuencia de talleres aislados y esporádicos. No se tienen en cuenta la forma en que el profesorado genera aprendizaje profesional. Darling Hammond (2017) llega a las mismas conclusiones y señala que la interacción y el intercambio influyen significativamente en cómo aprenden los profesores. Podemos, entonces, preguntarnos acerca de los formatos más adecuados para un aprendizaje docente basado en la colaboración y el apoyo mutuo.

Entre los múltiples ejemplos para impulsar la colaboración, aparece el caso de Japón, que tiene un sistema de comunidades de práctica, vinculado al estrategia que se ha denominado "Lesson study", donde los docentes planifican juntos y posteriormente desarrollan y analizan su enseñanza de form colaborativa. Los docentes visitan a sus pares, y las actividades de intercambio se centran en lograr que las buenas prácticas sean conocidas por el colectivo docente (Barber y Mourshed, 2007; Fox y Poultney, 2020).

En Chile, existen también múltiples ejemplos de comunidades de práctica y redes de colaboración. Tal es el caso de la Facultad de Educación de la Universidad Diego Portales donde se ha desarrollado un dispositivo de apoyo a la etapa final de la formación práctica, denominado Comunidades Virtuales de Aprendizaje (Lagos, 2016). Se trata de una metodología que busca favorecer el trabajo colaborativo entre docentes y que tiene en cuenta, para su implementación, la utilización de recursos tecnológicos como las plataformas virtuales o las filmaciones. 


\section{Quebrar la cultura reproductiva: Hacia el reconocimiento de los procesos de autoformación}

Una de las características de las profesiones en el siglo XXI es su permanente evolución y cambio. Los alumnos que ingresan hoy a la escuela van a desempeñarse en puestos de trabajos que probablemente no existen en la actualidad. Los centros educativos en algunos países también han comenzado a transformarse. ¿Dónde aprenden los docentes a prepararse y anticiparse a esos cambios? Evidentemente no en la formación inicial docente, bastante anclada en una cultura reproductiva. Es a través de la autoformación como algunos docentes encuentran la respuesta a las nuevas necesidades (Vaillant y Marcelo, 2015).

Aprendizaje informal, aprendizaje experiencial y procesos de autoformación están cada vez más presentes en la vida de maestros y profesores debido a las facilidades que brinda la tecnología y la posibilidad de conectarnos unos con otros. El proceso de aprendizaje autónomo permite la adquisición de habilidades y conocimientos pero, a su vez, plantea cuatro desafíos principales: conceptual, metodológico, de reconocimiento y pedagógico (Lecat et al., 2019).

El reto metodológico se relaciona con la necesidad de desarrollar estrategias innovadoras de investigación que permitan captar el conocimiento tácito en la formación docente. Es difícil para los investigadores aislar el conocimiento que adquieren los docentes mediante procesos informales, de sus prácticas rutinarias. Esto crea un doble desafío para la investigación. Por un lado, es difícil identificar procesos de aprendizaje que no son demasiado explícitos. Por otro lado, resulta complejo visibilizar los resultados del aprendizaje informal y descubrir nuevas habilidades de las que generalmente no somos conscientes. El aprendizaje informal involucra procesos cognitivos, emocionales y sociales por lo que la investigación no puede centrarse únicamente en la adquisición de conocimientos.

El desafío del reconocimiento se relaciona con la necesidad de mejorar los mecanismos institucionales para reconocer y evaluar los aprendizajes informales, experienciales y por autoformación. Históricamente, el aprendizaje informal no ha sido reconocido por las instituciones educativas, ya sea la escuela o la universidad. Esta situación ha comenzado a cambiar desde hace ya algún tiempo, con la aparición, de sistemas de evaluación y acreditación del aprendizaje experiencia (Kennedy, 2019).

Las aulas virtuales, las páginas web educativas, los blogs, las redes sociales, se han expandido entre los futuros docentes. En la actualidad, muchos de ellos emplean internet para el ocio o la comunicación personal, pero también como una herramienta educativa y de uso profesional. Las redes o comunidades virtuales de docentes han surgido y se han expandido en el ciberespacio (Marcelo y Marcelo, 2021). El confinamiento producido por el Covid-19, ha incrementado las modalidades de aprendizaje a distancia, mediante la utilización de una diversidad de formatos y plataformas (Maity et al., 2021; Schleicher, 2020).

Pero si ha habido en los tiempos recientes un espacio clave para la autoformación han sido los MOOCs (Cursos Masivos Online Abiertos). Se trata de una experiencia pionera por parte de las mejores universidades del mundo que poco a poco se ha ido extendiendo a centros universitarios de habla hispana. Se han creado plataformas específicas como Udacity, Coursera, EdX entre otras.

En el aprendizaje informal y la autoformación, el futuro profesor se prepara fuera de la institución educativa y de cualquier tipo de acompañamiento. Cada vez más esas modalidades rompen con la idea de aprendizaje en solitario por la presencia de las redes sociales (información en línea, blog, Twitter, Facebook ...). Los docentes en formación ya no están solos ya que comparten con otros. Y el futuro depara seguramente posibilidades infinitas con el surgimiento de la web 3.0 relacionada con la inteligencia artificial. Sitios web que se conectan entre sí según intereses del usuario y tecnologías de inteligencia artificial que vuelven la navegación más fluida y enriquecedora, ofrecerán nuevas oportunidades para el aprendizaje autónomo.

Los procesos de aprendizaje informal, de aprendizaje por la experiencia, de autoformación son una realidad desde hace décadas simplemente que hoy cobran nueva vigencia por la oportunidad que 
ofrece el ciberespacio. En ese sentido hay que entender a la formación como un fenómeno mucho más complejo que una serie de cursos presenciales. Incorporar el aprendizaje informal a las prácticas de formación y otorgarle reconocimiento supone un cambio de cultura de trabajo profesional. Implica también apostar por un modelo de formación comprometido con la auto regulación y la autonomía del docente.

Facebook, Instagram o Twitter se han convertido en espacios privilegiados en los que no sólo se encuentra la crítica, sino especialmente la construcción de comunidad de práctica en los espacios virtuales (Carpenter et al., 2019). Futuros maestros y profesores aprenden de manera informal a través de otros colegas. Nos referimos a profesionales de la educación (docentes, investigadores, escritores) que llevan su compromiso profesional más allá de sus espacios locales para convertirse en personas que generan "espacios de afinidad" (Rosenberg et al., 2016)some of the largest affinity spaces may be those organized around hashtags on Twitter: These spaces are public, largely unmoderated, and thriving, yet very little is known about them, especially those based in geographical areas such as American states. This paper examines these potential affinity spaces by providing the first large-scale study of them in the form of an examination of 47 State Educational Twitter Hashtags (SETHs. De acuerdo con Gee (2005), un espacio de afinidad es un "lugar donde las personas se afilian con otros basándose principalmente en actividades, intereses y objetivos compartidos" (p. 67). Estos espacios generan comunidades, ya no únicamente compuestas por temas o tópicos populares, sino por personas que buscan una conexión y colaboración entre ellos (Carpenter et al., 2020).

\section{A modo de conclusión: Las llaves para el cambio}

Resulta evidente la incidencia de la preparación de los maestros y de los profesores que hoy están en los centros de formación, en la vida de los niños y de los adolescentes de décadas futuras. Y, sin embargo, los modelos pedagógicos que sustentan la preparación inicial de maestros y profesores han perpetuado una formación caracterizada por el divorcio entre teoría y práctica y con un curriculum generalmente fragmentado. Es escaso el espacio para pensar la innovación, el cambio y la transformación de estrategias y metodologías.

El cambio que requiere la formación de maestros y profesores es drástico. No es suficiente con encarar meras 'reformas', sino que la profundidad de la necesaria transformación demanda una nueva visión acerca de cuál es el problema, cómo se lo define y cuáles son las alternativas posibles. El agotamiento de la propuesta tradicional nos ha llevado a plantear, en el presente artículo, la construcción de una visión alternativa. Es por esa razón que reflexionamos en "clave disruptiva" y sugerimos algunos caminos para cambiar el sustento pedagógico de la formación inicial docente. Hemos mencionado cuatro alternativas que a nuestro entender quiebran el modelo tradicional (Marcelo y Vaillant, 2018)

La primera alternativa disruptiva que proponemos refiere a la necesidad de romper con aulas tradicionales en las que reina la super especialización. Reclamamos el desarrollo de espacios de formación que permitan un aprendizaje profundo por parte de los estudiantes de formación docente. La orientación debería ser la integración y la interdisciplinaridad basada en "prácticas-clave"

Nuestra segunda sugerencia disruptiva, propone incorporar en la malla curricular de la formación, la reflexión acerca de las creencias y de los pensamientos de maestros y profesores. Reivindicamos la consideración del aspecto emocional de la docencia. Al enseñar generamos emociones y éstas necesariamente tienen que estar presentes en los procesos de formación inicial. Se trata de una reflexión que no surge por generación espontánea, sino que debemos alimentarla para poder indagar acerca de lo que siente y piensa el docente cuando está en el aula.

Promover estrategias que den paso a la colaboración e intercambio es nuestra tercera sugerencia disruptiva. La actividad docente necesita que los profesores estén en contacto, intercambien diferentes conocimientos, experiencias e innovaciones. En la formación inicial, la capacidad de intercambio y colaboración con otros, se debería potenciar en las propuestas curriculares. 
Por último, postulamos que los aprendizajes informales y los procesos de autoformación deberían tener un debido reconocimiento. Esas modalidades deben examinarse a la luz de las redes sociales y de la web 3.0. Hoy la tecnología ofrece nuevas posibilidades para el aprendizaje autónomo y para intercambiar con colegas de distintos puntos del planeta.

Tal como hemos intentado evidenciar en este artículo, existen experiencias disruptivas que transformaron la formación de docentes. Se trata de innovaciones que evidencian, entre otros un aprendizaje autónomo y colaborativo, con intercambio y con reconocimiento de los aspectos socioemocionales referidos a la tarea de enseñar lo que implica representa una verdadera mutación. El gran reto es cómo hacer que esas innovaciones y experiencias disruptivas sean escalables y una realidad para la formación inicial docente de las próximas décadas.

Nuestra intención al redactar este artículo ha sido ofrecer ideas que puedan inspirar la innovación en instituciones de formación inicial docente con particular interés por Iberoamérica que es la región en la que nos desempeñamos. Podrían aplicarse las cuatro propuestas como un todo o bien de forma parcial. Las innovaciones que hemos presentado han sido ya implementadas en contextos formativos particulares. Sin embargo, sería de interés que pudiéramos ampliar la base de conocimiento sobre sus resultados, mediante la descripción y evaluación no sólo a nivel de la implementación, sino de la incidencia a corto y medio plazo que generan tanto en los futuros docentes, como en los formadores que las apliquen.

\section{Referencias}

Aarts, R., Kools, Q. y Schildwacht, R. (2020). Providing a good start. Concerns of beginning secondary school teachers and support provided. European Journal of Teacher Education, 43(2), 277-295. https://doi.org/10.1080/02619768.2019.1693992

Adams, R., Kim, Y. M. y Greene, W. (2013). Actualizing core strengths in new teacher development. En Fred A. J. Korthagen, Y. M. Kim y W. L. Greene (Eds.), Teaching and learning from within a core reflection approach to quality and inspiration in education. Routledge.

Aguerrondo, I. y Vaillant, D. (2015). El aprendizaje bajo la lupa: Nuevas perspectivas para América Latina y el Caribe. UNICEF.

Attema-Noordewier, S., Korthagen, F. y Zwart, R. (2012). Promoting quality from within: A new perspective on professional development in schools. En M. Kooy y K. van Veen (Eds.) Teacher learning that matters: International perspectives (pp. 157-187). Routledge. https://doi.org/10.4324/9780203805879

Ávalos, B. (2014). La formación inicial docente en Chile: Tensiones entre políticas de apoyo y control. Estudios Pedagógicos, 40(e), 11-28 https://doi.org/10.4067/S0718-07052014000200002

Barber, M. y Mourshed M. (2007). How the world's best performing school systems come out on top. McKinsey \& Co.

Berry, A., Depaepe, F. y Van Driel, J. H. (2016). Pedagogical content knowledge in teacher education. En J. Loughran y M. Hamilton (Eds.), International handbook of teacher education (pp. 347-386). Springer.

Bolick, C. M., Glazier, J. y Stutts, C. (2019). Disruptive experiences as tools for teacher education: Unearthing the potential of experiential education. Journal of Experiential Education, 43(1), 21-36. https://doi.org/10.1177/1053825919877212

Boyle, B., While, D. y Boyle, T. (2004). A longitudinal study of teacher change: What makes professional development effective? The Curriculum Journal, 15(1), 45-68. https://doi.org/10.1080/1026716032000189471

Carpenter, J. P., Kimmons, R., Short, C. R., Clements, K. y Staples, M. E. (2019). Teacher identity and crossing the professional-personal divide on twitter. Teaching and Teacher Education, 81, 1-12. https://doi.org/https://doi.org/10.1016/j.tate.2019.01.011

Carpenter, J. P., Morrison, S. A., Craft, M. y Lee, M. (2020). How and why are educators using Instagram? Teaching and Teacher Education, 96, 103-149. https://oi.org/https://doi.org/10.1016/j.tate.2020.103149 
Christensen, C., Raynor, M. y McDonald, R. (2015). What is disruptive innovation? https://hbr.org/2015/12/what-is-disruptive-innovation

Daniels, H., Tse, H., Stables, A. y Cox, S. (2017). Design as a social practice: The design of new build schools. Oxford Review of Education, 43(6) 767-787. http://doi.org/10.1080/03054985.2017.1360176

Darling-Hammond, L. (2017). Teacher education around the world: What can we learn from international practice? European Journal of Teacher Education, 40(3), 291-309. https://doi.org/10.1080/02619768.2017. 1315399

Dinkelman, T. y Cuenca, A. (2020). A turn to practice: Core practices in social studies teacher education. Theory and Research in Social Education, 48(4), 583-610. https://doi.org/10.1080/00933104.2020.1757538

Dotger, B. (2015). Core pedagogy: Individual uncertainty, shared practice, formative ethos. Journal of Teacher Education, 66(3), 215-226. https://doi.org/10.1177/0022487115570093

Estola, E., Heikkinen, H. y Syrjälä, L. (2014). Narrative pedagogies for peer groups. En C. Craig y L. Orland-Barak (Eds.), International teacher education: Promising pedagogies (pp. 155-172). Emerald Group Publishing Limited.

Farrell, T. S. C. (2016). Surviving the transition shock in the first year of teaching through reflective practice. System, 61, 12-19. https://doi.org/10.1016/j.system.2016.07.005

Feiman-Nemser, S. (2001). From preparation to practice: Designing a continuum to strengthen and sustain teaching. Teachers College Record, 103, 1013-1055.

Fernández Enguita, M. (2017). Más escuela y menos aula. Morata.

Flores, A. (2016). Teacher education curriculum. En J. Loughran y M. Hamilton (Eds.), International handbook of teacher education (pp. 187-230). Springer.

Fox, A. y Poultney, V. (2020). Teacher professional learning through lesson study: Teachers' reflections. International Journal for Lesson and Learning Studies, 9(4), 397-412. https://doi.org/10.1108/IJLLS-03-2020-0011

Gee, J. P. (2005). Semiotic social spaces and affinity spaces. En D. Barton y K. Tusking (Eds.), Beyond communities of practice language power and social context (pp. 214-232). Cambridge University Press.

Gómez-Hurtado, I., Moya Maya, A. y García-Rodríguez, M. P. (2019). Aprendizaje servicio en la formación inicial de docentes en la Universidad de Huelva. REICE. Revista Iberoamericana sobre Calidad, Eficacia y Cambio en Educación, 18(1), 105-123. https://doi.org/10.15366/reice2020.18.1.006

Grosser-Clarkson, D. y Neel, M. A. (2020). Contrast, commonality, and a call for clarity: A review of the use of core practices in teacher education. Journal of Teacher Education, 71(4), 464-476.

https://doi.org/10.1177/0022487119880162

Grossman, P., Hammerness, K. y McDonald, M. (2009). Redefining teaching, re-imagining teacher education. Teachers and Teaching, 15(2), 273-289. http://doi.org/10.1080/135406009028753401

Hadar, L. y Brody, D. (2010). From isolation to symphonic harmony: Building a professional development community among teacher educators. Teaching and Teacher Education, 26(8), 1641-1651.

https://doi.org/10.1016/j.tate.2010.06.015

Hargreaves, A. (2005). Profesorado, cultura y postmodernidad. Cambian los tiempos, cambia el profesorado. Morata

Hoekstra, A., Beijaard, D., Brekelmans, M. y Korthagen, F. (2007). Experienced teachers' informal learning from classroom teaching. Teachers and Teaching: Theory and Practice, 13(2), 191-208.

https://doi.org/10.1080/13540600601152546

Howe, E. y Masahiro, A. (2014). Narrative teacher education pedagogies from across the Pacific. En C. Craig y L. Orland-Barak (Eds.), International teacher education: Promising pedagogies (pp. 213-232). Emerald Group Publishing Limited. https://doi.org/10.1108/S1479-368720140000022014

Janssen, F., Westbroek, H. y Doyle, W. (2014). The practical turn in teacher education: Designing a preparation sequence for core practice frames. Journal of Teacher Education, 65(3), 195-206.

https://doi.org/10.1177/0022487113518584 
Kelchtermans, G. (2014). Narrative-biographical pedagogies in teacher education. En C. Craig y L. Orland-Barak (Eds.), International teacher education: Promising pedagogies (pp. 273-291). Emerald Group Publishing Limited.

Kennedy, M. (2016). Parsing the practice of teaching. Journal of Teacher Education, 67(1), 6-17. https://doi.org/10.1177/0022487115614617

Kitchen, J. y Petrarca, D. (2016). Approaches to teacher education. En J. Loughran y L. Hamilton (Eds.), International handbook of teacher education (pp. 137-185). Springer.

Kleickmann, T., Richter, D., Kunter, M., Elsner, J., Besser, M., Krauss, S. y Baumert, J. (2013). Teachers' content knowledge and pedagogical content knowledge: The role of structural differences in teacher education. Journal of Teacher Education, 1, 90-106. https://doi.org/10.1177/0022487112460398

Kennedy, M. (2019). How we learn about teacher learning. Review of Research in Education, 43(1), 138-162. https://doi.org/10.3102/0091732X19838970

Korthagen, F. (2014). Promoting core reflection in teacher education: Deepening professional growth. En C. Craig y L. Orland-Barak (Eds.), International teacher education: Promising pedagogies (pp. 73-90). Emerald Group Publishing Limited.

Korthagen, F. (2016). Pedagogy of teacher education. En J. Loughran y M. Hamilton (Eds.), International handbook of teacher education (pp. 311-346). Springer.

Korthagen, F. y Nuijten, E. E. (2018). Core reflection: Nurturing the human potential in students and teachers. En J. Miller, K. Nigh, Marni J. Binder, B. Novak y S. Crowell (Eds.), International handbook of holistic education (pp. 84-95). Routledge. https://doi.org/10.4324/9781315112398

Kyndt, E., Gijbels, D., Grosemans, I. y Donche, V. (2016). Teachers' everyday professional development: Mapping informal learning activities, antecedents, and learning outcomes. Review of Educational Research, 86(4), 1111-1150. https://doi.org/10.3102/0034654315627864

Lagos, C. (2016). Experiencias de innovación en la formación práctica de carreras de pedagogía en Chile. Organización de Estados Iberoamericanos.

Lampert, M., Franke, M., Kazemi, E., Ghousseini, H., Turrou, A., Beasley, H. y Crowe, K. (2013). Keeping it complex: Using rehearsals to support novice teacher learning of ambitious teaching. Journal of Teacher Education, 64, 226-243. https://doi.org/10.1177/0022487112473837

Lecat, A., Raemdonck, I., Beausaert, S. y März, V. (2019). The what and why of primary and secondary school teachers' informal learning activities. International Journal of Educational Research, 96, 100-110. https://doi.org/10.1016/j.ijer.2019.06.003

Loh, J. (2014). The narrative as the practical and the practice: Toward the preparation of teachers. En C. Craig y L. Orland-Barak (Eds.), International teacher education: Promising pedagogies (pp. 195-212). Emerald Group Publishing Limited.

Lortie, D. C. (1975). Schoolteacher: A sociological study. University of Chicago Press.

Lunenberg, M., Dengerink, J. y Korthagen, F. (2014). The professional teacher educator: Roles, behaviour, and professional development of teacher educators. Sense Publishers. https://doi.org/10.1007/978-94-6209-518-2

Maity, S., Sahu, T. N. y Sen, N. (2021). Panoramic view of digital education in COVID-19: A new explored avenue. Review of Education, 9(2), 405-423. https://doi.org/10.1002/rev3.3250

Marcelo, C. y Marelo, P. (2021). Educational influencers on twitter. Analysis of hashtags and relationship structure. Comunicar, 29(68), 71-80. https://doi.org/10.3916/C68-2021-06

McDonald, M., Kazemi, E. y Schneider Kavanagh, S. (2013). Core practices and pedagogies of teacher education: A call for a common language and collective activity. Journal of Teacher Education, 64(5), 378-386. https://doi.org/10.1177/0022487113493807

McGraw, A. (2014). Disperdes narratives and powerful teacher education pedagogy. En C. Craig y L. Orland-Barak (Eds.), International teacher education: Promising pedagogies (pp. 173-193). Emerald Group Publishing Limited. 
Mérida-Serrano, R., González-Alfaya, M. E., Olivares-García, M. de los Ángeles, Rodríguez-Carrillo, J. y MuñozMoya, M. (2020). El prácticum, un espacio para la investigación transformadora en los contextos educativos infantiles. REICE. Revista Iberoamericana sobre Calidad, Eficacia y Cambio en Educación, 18(2), 17-34. https://doi.org/10.15366/reice2020.18.2.001

Murillo Arango, G. (2015). Narrativas de experiencia en educación y pedagogía de la memoria. Editorial de la Facultad de Filosofía y Letras Universidad de Buenos Aires.

OCDE. (2019). TALIS 2018 Results. Teachers and school leaders as lifelong learners. OCDE.

Patton, K. y Parker M. (2014). Teacher education communities of practice: More than a culture of collaboration. Teaching and Teacher Education 67, 351-360. https://doi.org/10.1016/j.tate.2017.06.013

Rosenberg, J. M., Greenhalgh, S. P., Koehler, M. J., Hamilton, E. R. y Akcaoglu, M. (2016). An investigation of state educational twitter hashtags (SETHs) as affinity spaces. E-Learning and Digital Media, 13(2), 24-44. https://doi.org/10.1177/2042753016672351

Russell, T. (2014). One teacher educator's career-long development of pedagogy of reflection. En C. Craig y L. Orland-Barak (Eds.), International teacher education: Promising (pp. 55-72). Emerald Group Publishing Limited.

Russell, T. (2018). A teacher educator's lessons learned from reflective practice. European Journal of Teacher Education, 41(1), 4-14. https://doi.org/10.1080/02619768.2017.1395852

Schön, D. (1983). The reflective practitioners: How professionals think in Action. Basic Books.

Schleicher, A. (2020). The impact of Covid-19 on education insights from education at a glance 2020. OECD.

Stoll, L. y Louis. K. S. (2007). Professional learning communities: divergence, depth and dilemmas. McGraw Hill.

Van Dijk, E. M. (2008). Teachers' views on understanding evolutionary theory: A PCK-study in the framework of the ERTE-model. Teaching and Teacher Education, 25(2) 259-267.

Ward, P. (2021). Core practices for teaching physical education: Recommendations for teacher education. Journal of Teaching in Physical Education, 4O(1), 98-108. https://doi.org/10.1123/JTPE.2019-0114

Wolfswinkel, J., Furtmueller, E. y Wilderom, C. (2013). Using grounded theory as a method for rigorously reviewing literature. European Journal of Information Systems, 22(1), 45-55. https://doi.org/10.1057/ejis.2011.51

Zavelevsky, E. y Lishchinsky, O. S. (2020). An ecological perspective of teacher retention: An emergent model. Teaching and Teacher Education, 88, 102965. https://doi.org/10.1016/j.tate.2019.102965

\section{Breve CV de los/as autores/as}

\section{Denise Vaillant}

Directora del Programa de Doctorado en Educación de la Universidad ORT Uruguay. Directora de la revista Cuadernos de Investigación Educativa. Profesora invitada en numerosas universidades latinoamericanas y europeas, asesora de varios organismos internacionales y autora de artículos y libros referidos a la temática de políticas docentes e innovación educativas. Coordina programas de investigación en el campo de su especialidad y es Investigadora Nivel III del Sistema Nacional de Investigadores (SNI) de Uruguay. Integra numerosos consejos directivos de revistas académicas, organismos y fundaciones vinculadas con el campo educativo entre los cuales el Consejo de Administración de la Fundación CEIBAL. Email: vaillant@ort.edu.uy

ORCID ID: https://orcid.org/0000-0001-8110-4634

\section{Carlos Marcelo}

Catedrático de Universidad en la Universidad de Sevilla. Actualmente cuenta con 7 sexenios de investigación. Según Google Scholar, con un índice h 55. Premio a la Trayectoria Investigadora de Excelen- 
cia por Ciencias Sociales de la Universidad de Sevilla en 2016. Dirige el Grupo de Investigación IDEA, grupo de excelencia. Fue miembro del equipo de expertos internacionales de la OEI (Organización de Estados Iberoamericanos) en Formación Docente en Programa de Metas 2020. Ha sido asesor de organismos internacionales. Su especialización ha sido la formación docente, campo donde ha publicado libros y artículos. Email: marcelo@us.es

ORCID ID: https://orcid.org/0000-0002-8547-367X 